\title{
Primary or secondary antifungal prophylaxis in patients with hematological maligancies: efficacy and damage
}

This article was published in the following Dove Press journal:

Therapeutics and Clinical Risk Management

28 April 2014

Number of times this article has been viewed

\author{
Habip Gedik' \\ Funda Șimșek' \\ Taner Yıldırmak' \\ Arzu Kantürk' \\ Deniz Arıca ${ }^{2}$ \\ Demet Aydın² \\ Naciye Demirel ${ }^{2}$ \\ Osman Yokuș² \\ 'Department of Infectious \\ Diseases and Clinical Microbiology, \\ ${ }^{2}$ Department of Hematology, Ministry \\ of Health Okmeydanı Training and \\ Research Hospital, İstanbul, Turkey
}

Correspondence: Habip Gedik

Department of Infectious Diseases and

Clinical Microbiology, Ministry of Health

Okmeydanı Training and Research

Hospital, SB Okmeydanı Eğitim ve

Araștırma Hastanesi Șișli-Istanbul, Turkey

Tel +902123145555

Fax +902122217800

Email habipgedik@yahoo.com
Background: Patients with hematological malignancies often develop febrile neutropenia (FN) as a complication of cancer chemotherapy. Primary or secondary antifungal prophylaxis is recommended for patients with hematological malignancies to reduce the risk of invasive fungal infection (IFI). This study retrospectively evaluated the efficacy and potential harm of administration of primary and secondary antifungal prophylaxis to patients with hematological malignancies at one hospital.

Methods: All patients with hematological malignancies older than 14 years of age who had experienced at least one FN attack during chemotherapy while being treated at one hospital between November 2010 and November 2012 were retrospectively evaluated.

Results: A total of $282 \mathrm{FN}$ episodes in 126 consecutive patients were examined during a 2 -year study period. The mean patient age was $51.73 \pm 14.4$ years (range: $17-82$ years), and 66 patients were male. Primary prophylaxis with posaconazole was administered to 13 patients and systemic antifungal treatment under induction or consolidation chemotherapy to seven patients. Of 26 patients who received secondary antifungal prophylaxis with either oral voriconazole $(n=17)$ or posaconazole $(n=6)$ during 46 FN episodes, systemic antifungal therapy was administered in 16 of 38 episodes and three of eight episodes, respectively. Secondary antifungal prophylaxis with caspofungin was found effective in treating six FN episodes in three patients who had experienced at least two persistent candidemia attacks. The mortality rates associated with IFI were $9 \%$ in the first year, $2 \%$ in the second year, and $6 \%$ overall. The mortality rates associated with candidemia were $33 \%$ in the first year, $22 \%$ in the second year, and $27 \%$ overall.

Conclusion: Primary antifungal prophylaxis should be administered to selected patients on the basis of consideration of efficacy, cost, and potential harm. Use of secondary prophylaxis may reduce systemic antifungal use and IFI frequency but may increase risk of colonization and infection with azole-resistant fungal strains.

Keywords: azole resistance, febrile neutropenia, hematological malignancy, invasive fungal infection, primary antifungal prophylaxis, secondary antifungal prophylaxis

\section{Introduction}

Patients with hematological malignancies often develop febrile neutropenia (FN) as a complication of cancer chemotherapy. ${ }^{1}$ Opportunistic infection, especially invasive fungal infection (IFI), is often observed during the neutropenic phase in these patients. Among patients who develop infection, invasive Aspergillus infection has been observed in patients at risk due to prolonged and profound neutropenia related to hematologic malignancy or allogeneic hematopoietic stem cell transplant. ${ }^{2}$ Antifungal prophylaxis using an antifungal drug such as fluconazole (FLC), itraconazole (ITR), 
voriconazole (VOR), posaconazole (POS), micafungin, and/ or caspofungin (CAS) is thus recommended for patients with hematological malignancies who undergo a hematopoietic stem cell transplant or intensive remission-induction or salvage-induction chemotherapy for acute leukemia. ${ }^{1}$ Specifically, antifungal prophylaxis is recommended for selected patients who are over 13 years of age and at high risk of invasive Aspergillus infection because they are undergoing intensive chemotherapy for acute myeloblastic leukemia (AML) or myelodysplastic syndrome, induction for acute leukemia, or a preengraftment allogeneic hematopoietic stem cell transplant. ${ }^{1}$ Use of antifungal prophylaxis with POS has been reported to reduce overall costs compared with use of either FLC or ITR. ${ }^{3}$ The use of primary antifungal prophylaxis has been subjected to more debate than that of secondary antifungal prophylaxis in terms of costs, efficacy, and harm. ${ }^{3}$ This study retrospectively evaluated the efficacy and potential harm of use of primary and secondary antifungal prophylaxis in patients with hematological malignancies.

\section{Materials and methods Study population}

All subjects included in this study had been treated for hematological cancer in the hematology ward of the Ministry of Health Okmeydani Training and Research Hospital in Istanbul between November 2010 and November 2012. The inclusion criteria were age over 14 years and experience of at least one FN episode after chemotherapy. The exclusion criterion was treatment for other hematological diseases, such as anemia or idiopathic or immune thrombocytopenic purpura. This study was approved by the local ethics committee $(\mathrm{MoH}$ Okmeydanı Training and Research Hospital). The patient rooms in the 23-bed hematology ward contain either one, two, or four beds and three shared bathrooms and do not have high-efficiency particulate air (HEPA) filters. The patients and their attendants remained in the same room and used the shared facilities. FN was diagnosed if the patient's oral temperature was either $38.3^{\circ} \mathrm{C}$ or higher at one measurement or higher than $38.0^{\circ} \mathrm{C}$ for at least 2 hours at two consecutive measurements and absolute neutrophil count was either less than or anticipated to fall below $0.5 \times 10^{9} / \mathrm{L} .{ }^{1}$ Data regarding patient demographics, diagnosis, clinical episodes, clinical presentation and therapy, laboratory and microbiological findings, hematological malignancies, and outcome were collected from hospital records. The Multinational Association of Supportive Care in Cancer (MASCC) prognostic index was used to evaluate the severity of FN. ${ }^{1}$

\section{Prophylaxis and diagnosis of IFI}

The primary and secondary antifungal prophylaxis protocols described in the Clinical Practice Guidelines for the Use of Antimicrobial Agents in Neutropenic Patients with Cancer by the Infectious Diseases Society of America in 2002 and subsequently updated in 2010 were followed in the hematology ward. ${ }^{1,4}$ Patients with a risk of invasive Candida infection and/or invasive Aspergillus infection or who were undergoing intensive remission-induction or salvage-induction chemotherapy for acute leukemia received antifungal prophylaxis in accordance with the guidelines. POS for primary antifungal prophylaxis was administered orally in a dose of $200 \mathrm{mg}$ three times per day with a fat-containing meal and acidic fruit juice. Secondary antifungal prophylaxis was administered as either $200 \mathrm{mg}$ of oral VOR twice per day or $200 \mathrm{mg}$ of oral POS three times per day. Possible invasive pulmonary Aspergillus (IPA) infection was defined as clinical and radiologic findings highly suggestive of infection without established histopathologic and/or microbiologic evidence of infection. Probable IPA infection was defined as a positive culture for Aspergillus species from a respiratory specimen or two consecutive Aspergillus galactomannan antigen tests with an index of $\geq 0.5$ supported by clinical and radiologic findings. Proven IPA infection was defined as histopathologic evidence of tissue invasion and damage by Aspergillus species supported by clinical and radiologic findings. ${ }^{5}$ CAS for treatment and prophylaxis was administered by intravenous infusion at an initial dose of $70 \mathrm{mg}$, followed by intravenous infusion at a dose of $50 \mathrm{mg}$ per day. Blood cultures were performed by inoculation into BactAlert 3D bottles (BioMérieux, Craponne, France). Urine, sputum, wound, conjunctive, abscess, and catheter samples were inoculated onto 5\% sheep blood agar (Salubris Inc., Istanbul, Turkey), chocolate agar (Salubris Inc.), and MacConkey agar (Salubris Inc.) for microbiological analysis. Yeast isolated from blood cultures was identified by morphologic examination on Sabouraud dextrose agar plates (Unipath Ltd, Basingstoke, UK). Examination of germ-tube formation and API ID 32C yeast identification system (BioMérieux) was performed for discrimination at the species level. Susceptibility testing according to the Clinical and Laboratory Standards Institute broth microdilution M27-A2 protocols for Candida species and the M38-A protocols for Aspergillus species was performed using the ATB Fungus 2 microdilution kit (BioMérieux) with $0.03-64 \mathrm{mg} / \mathrm{L}$ of 5-fluorocytosine, $0.008-16 \mathrm{mg} / \mathrm{L}$ of amphotericin B, 0.125-256 mg/L of FLC, and $0.008-16 \mathrm{mg} / \mathrm{L}$ of ITR. VOR sensitivity testing was performed using the E test (AB Biodisk, Solna, Sweden). Candida krusei American Type Culture Collection (ATCC) 
14243, Candida parapsilosis ATCC 22019, and Aspergillus fumigatus ATCC 204305 were used as reference strains.

Aspergillus galactomannan antigen testing was performed using the Platelia Aspergillus EIA commercial enzyme immunoassay kit (Bio-Rad Laboratories, Hercules, CA, USA). Blood samples of patients were analyzed twice weekly, and the results were computed as an index in which values of $\geq 0.5$ relative to the optical density of the control sample measured with a semiautomatic analyzer (Behring ELISA processor III; Siemens, Munich, Germany) were considered positive samples. ${ }^{6}$ Possible causes of false positive and negative results were eliminated, taking into account the reasons that caused them. The test results were considered significant if two consecutive Aspergillus galactomannan antigen test results were computed as an index of $\geq 0.5$, under persistent or recurrent fever, after 4 to 7 days of antibiotic administration, either with or without microbiologic or radiological findings associated with IFI, and the overall duration of neutropenia was expected to be more than 7 days. ${ }^{1}$ VOR was administered in an initial loading dose of $6 \mathrm{mg} / \mathrm{kg}$ every 12 hours (q12h) intravenously for the first 24 hours and then in a maintenance dose of either $4 \mathrm{mg} / \mathrm{kg}$ q12h intravenously or $200 \mathrm{mg} \mathrm{q} 12 \mathrm{~h}$ orally for treatment of IFI. Administration of liposomal amphotericin B was initiated for treatment of IFI at a dose of $3.0 \mathrm{mg} / \mathrm{kg} /$ day and increased as required. Doses of antifungal drugs were adjusted according to the relevant guidelines, recommendations of pharmaceutical manufacturers, and condition of the patient. Nephrotoxicity was defined as an increase in serum creatinine value to over twice the baseline value or peaking at $>2.0 \mathrm{mg} / \mathrm{dL}$. Hypokalemia was defined as a serum potassium level $<3.5 \mathrm{mmol} / \mathrm{L}$ $(<3.5 \mathrm{mEq} / \mathrm{L})$. Moderate hypokalemia was defined as a serum potassium level of $2.5-3 \mathrm{mmol} / \mathrm{L}(2.5-3 \mathrm{mEq} / \mathrm{L})$ and severe hypokalemia as a serum potassium level $<2.5 \mathrm{mmol} / \mathrm{L}$ $(<2.5 \mathrm{mEq} / \mathrm{L}){ }^{7}$ Blood concentration of VOR could not be monitored owing to the inability to conduct an analysis in Turkey. Response to treatment was defined as defervescence in the 48-72 hours subsequent to the initiation of antimicrobial therapy, recovery of laboratory findings, and clinical symptoms associated with infected organ or system. The primary consequence was defined as in-hospital mortality during the neutropenic phase and the clinical outcomes as FN episodes.

\section{Statistical analysis}

Continuous variables were represented as the mean \pm standard deviation and the range. Percentile values were represented without decimals. Rates of systemic antifungal use were com- pared using the Pearson $\chi^{2}$ test. Statistical significance was defined as values reaching a $P<0.05$ level of significance. Overall mortality associated with FN was defined as death within 30 days of the development of FN. Crude 30-day mortality rates were calculated as the proportion of study patients who died within 30 days of FN development. The mortality rates of IFI cases were calculated as the proportion of patients who died of IFI within 30 days of the development of FN. Overall mortality rates of candidemia cases were calculated as the proportion of study patients who died of a candidemia attack within 30 days of the development of FN.

\section{Results}

A total of 282 febrile episodes were retrospectively analyzed in 126 consecutive patients with neutropenia over a 2-year study period, with 65 cases examined in the first year and 78 in the second year. The mean patient age was $51.73 \pm 14.4$ years (range: 17-82 years), and 66 patients were male. The mean MASCC score was $17.18 \pm 8.27$ in patients with hematological malignancies (Table 1). The mean duration of FN was $29.38 \pm 6.95$ days. Primary POS prophylaxis was administered during only the second year owing to unavailability of POS during the first year. Of the 13 patients to whom POS was administered, one patient with colostomy could not tolerate it because of diarrhea. Only seven (53\%) patients required systemic antifungal treatment under induction or consolidation chemotherapy. Of the 26 patients who received secondary antifungal prophylaxis, either via oral VOR ( $n=17)$ or POS $(n=6)$ over 46 episodes, systemic antifungal therapy was administered in 16 of 38 (42\%) and three of eight (37\%) FN episodes. Rates of systemic antifungal use were found to be similar in the POS-administered group and the VOR-administered group $(P>0.05)$. Secondary antifungal prophylaxis with CAS was found effective in

Table I Distribution of hematologic malignancies in patients with febrile neutropenia $(n=126)$

\begin{tabular}{ll}
\hline Hematologic malignancies & $\mathbf{n ~ ( \% )}$ \\
\hline Acute myeloblastic leukemia & $73(58)$ \\
Acute lymphocytic leukemia & $22($ I7) \\
Non-Hodgkin lymphoma & $7(5)$ \\
Chronic lymphocytic leukemia & $5(4)$ \\
Multiple myeloma & $5(4)$ \\
Hairy cell leukemia & $4(3)$ \\
Aplastic anemia & $3(2)$ \\
Chronic myeloid leukemia & $2(2)$ \\
Plasma cell leukemia & $2(2)$ \\
Mantle-cell lymphoma & $2(2)$ \\
Chronic lymphocytic leukemia with Burkitt's lymphoma & $\mathrm{I}(\mathrm{I})$ \\
Total & $\mathrm{I} 26(\mathrm{I00})$ \\
\hline
\end{tabular}


treating six episodes experienced by three patients who had experienced at least two persistent candidemia attacks. POS was discontinued because of diarrhea $(n=2,10 \%)$ or elevated liver enzymes $(n=1,5 \%)$ in 19 patients who received POS as primary or secondary prophylaxis therapy. VOR was discontinued due to hallucination accompanied with blurred and colored visual change $(n=1,5 \%)$ or elevated liver enzymes $(n=1,5 \%)$ in 17 patients. Blood concentration of VOR could not be monitored owing to the unavailability of analytical tools in Turkey.

No CAS-related side effects were reported. The distribution of IFI cases that were diagnosed and treated was 23 cases with culture-proven IFI in 31 FN attacks, 19 cases with probable IPA infection in $25 \mathrm{FN}$ attacks, 38 cases with possible IPA infection in $42 \mathrm{FN}$ attacks, 30 cases with suspected IFI in 31 attacks, and five cases (5\%) with hepatosplenic candidiasis over the 2-year period. Of the culture-proven IFI in 31 attacks in 23 cases, 22 attacks in 15 cases occurred in the first year and nine attacks in eight cases in the second year (Table 2). No evidence of infection associated with Zygomycetes or Fusarium species was obtained. Previous azole exposure was recorded in 13 of 18 cases (72\%) with bloodstream yeast infections. VOR and FLC resistance were identified in Candida parapsilosis, Candida glabrata, and Candida albicans isolates (Table 2). Overall 30-day crude mortality rates were $35 \%(23 / 65)$ in patients diagnosed with AML $(n=16)$, acute lymphocytic leukemia (ALL) $(n=5)$, multiple myeloma $(n=1)$, or chronic myeloid leukemia $(n=1)$ in the first year and $21 \%(17 / 78)$ in patients diagnosed with $\operatorname{AML}(n=16), \operatorname{ALL}(n=4)$, or non-Hodgkin lymphoma $(n=1)$ in the second year. Infectious complications contributed to death in 17 cases $(26 \%)$ in the first year, eleven cases $(14 \%)$ in the second year. The mortality rates associated with IFI were $9 \%(n=6)$ in the first year, $2 \%(n=2)$ in the second year, and $6 \%(\mathrm{n}=8)$ overall (Table 2$)$. The mortality rates associated with candidemia were 33\% (3/9 attacks) in the first year, 22\% (2/9 attacks) in the second year, and $27 \%$ $(5 / 18$ attacks $)$ overall.

\section{Discussion}

Primary antifungal prophylaxis with POS did not provide the expected benefit in the patients examined in the current study, with 53\% experiencing breakthrough IFI. In comparison, four previous studies reported a breakthrough IFI incidence of $3 \%$, $13 \%, 18 \%$, and $23 \%,{ }^{8-11}$ with one reporting the incidence ranging from $0 \%$ to $5 \%$ under primary prophylaxis with POS. ${ }^{10}$ The discrepancy between the findings of this study and previous studies may be attributable to the conditions in the hematology ward and the small number of cases examined, both of which may have increased the risk of breakthrough IFI in the patients who had received primary antifungal prophylaxis with POS compared to patients in other studies. A previous study reported a rate of yeast colonization of $21 \%$ as well as the emergence of rare strains of yeasts, but no strain of mold could be identified. ${ }^{9}$ Another study reported no improvement in mortality rates or increase in 90-day survival rates in patients who received primary antifungal prophylaxis with POS. ${ }^{10}$ These findings indicate that the cost-effectiveness and risk of colonization with azole-resistant fungal strains should be taken into consideration when primary antifungal prophylaxis with POS is administered. Another factor that should be considered is the hospital environment, as antifungal prophylaxis is not recommended in settings that have a relatively high incidence of invasive mold infection and do not have facilities for early diagnosis and treatment. ${ }^{12} \mathrm{We}$ suggest that primary antifungal prophylaxis cannot be useful in our hematology ward and other settings that do not have HEPA filters in the patients' rooms and lack certain tests for early diagnosis and treatment.

In the current study, most patients who had undergone primary antifungal prophylaxis with POS had AML, had a mean MASCC score $<21$, and had had FN for a mean duration of $29.38 \pm 6.95$ days, factors associated with a higher risk of IFI. In a study presented at the European Congress of Clinical Microbiology and Infectious Diseases in 2012, IFI developed in two of 24 patients who had received primary antifungal prophylaxis with POS and one of 24 patients who had received primary antifungal prophylaxis with oral FLC. ${ }^{13}$ Previously, invasive Aspergillus infection has been associated with relapse and mortality in immunocompromised patients, with one study showing patients with invasive Aspergillus relapse to have a mortality rate $88 \%-100 \%$ higher than that of patients with primary invasive Aspergillus. ${ }^{14}$ Another study found that secondary antifungal prophylaxis with either VOR or POS reduced systemic antifungal drug use and cost in 23 patients with AML in 27 of 46 (58\%) attacks. Secondary prophylaxis with VOR has also been reported effective in patients with acute leukemia. ${ }^{15}$ We have found secondary prophylaxis more effective than primary prophylaxis in terms of using systemic antifungal therapy in spite of limitations of the study. In a study looking at primary antifungal prophylaxis with either VOR, POS, CAS, or ITR, partial clinical response to prior invasive Aspergillus treatment, incomplete resolution of imaging findings before additional chemotherapy, use of systemic corticosteroids, lack of remission of the underlying of hematological malignancy, 


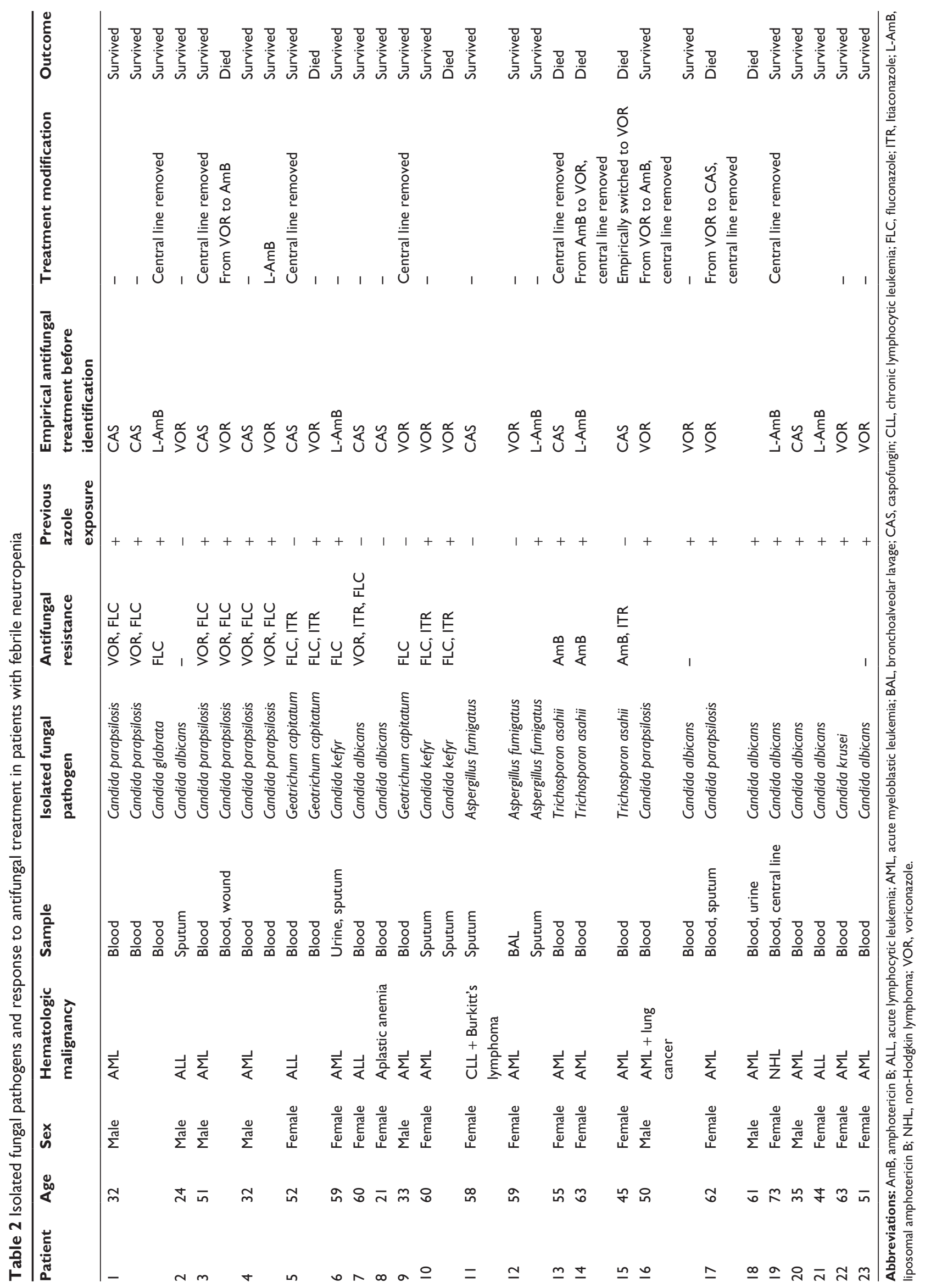


administration of more than three antibiotics, duration of neutropenia longer than 28 days, duration of primary antifungal therapy less than 1 month, and use of high-dose cytosine arabinoside were identified as factors predisposing to invasive Aspergillus relapse. ${ }^{16}$ These findings indicate that administration of secondary antifungal prophylaxis may be acceptable as primary prophylaxis, as laminar flow and use of a HEPA filter in the patient rooms can prevent reinfection with Aspergillus but not relapse. ${ }^{16}$

Most studies of secondary antifungal prophylaxis examined the use of amphotericin B, ITR, or their combined use in small patient groups. ${ }^{16}$ VOR was reported to be inappropriate in $40 \%$ of patients who had followed targeted, empirical, preemptive prophylactic regimens. ${ }^{17}$ As VOR has nonlinear pharmacokinetics, changes in serum level should be monitored in patients undergoing VOR therapy. ${ }^{18}$ POS and VOR were well tolerated when used as primary and secondary antifungal prophylaxis in the patients in the current study. Although POS is recommended for primary prophylaxis, it provided effective secondary prophylaxis in the patients who could not tolerate VOR in the current study. In a previous study, the complete and partial response rates were found to be significantly higher in 30 of 37 patients whose blood level of VOR had been monitored compared to 20 of 34 patients whose blood level of VOR had not been monitored (81\% versus $57 \%$, respectively). ${ }^{19}$ The study also found that the incidence of adverse events were not significantly different between the VOR-monitored and non-VOR-monitored patients. As was observed in the current study, hallucinations, elevated liver enzymes, and visual disturbances have been identified as important side effects of VOR, with the incidence of hallucinations and discontinuation due to elevated liver enzymes reported to range between $4.3 \%$ and $9 \%$ and between $4 \%$ and $8 \%$, respectively. ${ }^{20}$ Genetic polymorphism of the CYP2C19 isoenzyme, which is known to have a role in VOR metabolism, has been found to affect the serum level of VOR. The incidence of poor metabolism, which may lead the VOR level to be four times higher in those affected compared to those not affected, has been reported to be $20 \%$ in non-Indian Asians and $5 \%$ in Caucasians and African-Americans. ${ }^{21}$ Use of CYP2C19 isoenzyme inhibitors, such as omeprazole, and inducers, such as rifampin and phenytoin, should also be taken into consideration during VOR therapy. ${ }^{22}$ Fortunately, hepatotoxicity is reversible after discontinuation or reduction of dosage of VOR. ${ }^{23}$ The incidence of hallucinations in the current study was found to be higher than those previously reported..$^{22,24}$
The discontinuation rates of liposomal amphotericin B due to nephrotoxicity have been reported as $12.3 \%$ at a dose of $3 \mathrm{mg} / \mathrm{kg} /$ day (85 of 691 patients), 32.1\% at a dose of $5 \mathrm{mg} / \mathrm{kg} /$ day (81 of 252 patients), and $4.2 \%$ (17 of 406 patients). ${ }^{7,25}$ The incidence of hypokalemia as a side effect of liposomal amphotericin B has been reported as $6.2 \% .{ }^{24,25}$ POS-related hepatotoxicity has been reported as $3 \%$ and $3.7 \%$, rates comparable to the $4 \%$ found in the current study. ${ }^{26}$ Despite previous reports that CAS is ineffective as primary antifungal prophylaxis in patients with hematological malignancies, it may be effective for secondary prophylaxis as used in our study. ${ }^{8}$

Azole resistance emerged among Candida parapsilosis and Candida albicans isolated from the patients in the current study. The incidence of previous azole exposure in these patients indicated the existence of drug resistance (Table 2). Use of VOR, the most commonly administered antifungal drug, may have predisposed the patients to colonization with azole-resistant fungal pathogens. In accordance with this finding, the emergence of VOR resistance has been recently reported to be more prevalent than that of FLC resistance, reportedly ranging from $0.3 \%$ to $13.3 \%{ }^{27,28}$ Administration of either amphotericin B or CAS is recommended for treatment of azole-resistant Candida species-related bacteremia. ${ }^{29}$ In the current study, history of antifungal use was found to be associated with azoleresistant Candida species in patients with hematological malignancies (Table 2), indicating that antifungal drug use leads to development of relevant antifungal drug-resistant strains in the environment and patients. ${ }^{1,30}$ To address this problem, periodic changes in the use of antifungal drugs, such as changing VOR to amphotericin B for invasive Aspergillus and CAS to amphotericin B for empirical antifungal treatment, could be evaluated, but performance of prospective, randomized-controlled studies is needed to identify a solution to this challenge.

Although the blood concentration of the drugs examined in the current study could not be monitored, monitoring of drug concentration has been found to increase efficacy and decrease toxicity risk. The blood concentration of VOR has been reported not to reach therapeutic levels in approximately $50 \%$ of all patients. ${ }^{18}$ Another challenge regarding use of oral drugs is the development of azole resistance in Aspergillus and Candida species and related infections. ${ }^{31}$ Either amphotericin B or CAS may be used for treatment if IFI develops during or after treatment with another drug. ${ }^{32}$ Use of CAS has been reported ineffective as the primary antifungal prophylaxis in patients with hematological malignancies but 
could be effective for secondary prophylaxis as used in the current study. ${ }^{8}$ Prolonged use of CAS for prophylaxis has been found to predispose to colonization with resistant fungal pathogens that precede IFI in patients with hematological malignancies. ${ }^{33}$

This study faced several limitations that should be considered when reviewing the findings. The main limitation was use of a retrospective rather than a prospective and randomized design, which did not allow for determination of causal relationships. Another limitation was the small sample size, which may have limited the ability to evaluate the efficacy of primary antifungal prophylaxis appropriately. Further studies overcoming these limitations should focus on evaluating primary and secondary antifungal prophylaxis use in terms of benefits and disadvantages. While secondary prophylaxis may reduce systemic antifungal use and frequency of IPA infection, the fact that antifungal prophylaxis is predisposing to colonization and infection with azole-resistant fungal strains must remain a constant consideration.

\section{Disclosure}

The authors have no conflicts of interest to report in regard to this work.

\section{References}

1. Freifeld AG, Bow EJ, Sepkowitz KA, et al. Clinical practice guideline for the use of antimicrobial agents in neutropenic patients with cancer: 2010 update by the infectious diseases society of america. Clin Infect Dis. 2011;52(4):e56-e93.

2. de Naurois J, Novitzky-Basso I, Gill MJ, Marti FM, Cullen MH, Roila F; ESMO Guidelines Working Group. Management of febrile neutropenia: ESMO Clinical Practice Guidelines. Ann Oncol. 2010;21 Suppl 5: v252-v256.

3. Collins CD, Ellis JJ, Kaul DR. Comparative cost-effectiveness of posaconazole versus fluconazole or itraconazole prophylaxis in patients with prolonged neutropenia. Am J Health Syst Pharm. 2008;65(23): 2237-2243.

4. Hughes WT, Armstrong D, Bodey GP, et al. 2002 guidelines for the use of antimicrobial agents in neutropenic patients with cancer. Clin Infect Dis. 2002;34(6):730-751.

5. Ascioglu S, Rex JH, de Pauw B, et al; Invasive Fungal Infections Cooperative Group of the European Organization for Research and Treatment of Cancer; Mycoses Study Group of the National Institute of Allergy and Infectious Diseases. Defining opportunistic invasive fungal infections in immunocompromised patients with cancer and hematopoietic stem cell transplants: an international consensus. Clin Infect Dis. 2002;34(1):7-14

6. Maertens J, Theunissen K, Verbeken E, et al. Prospective clinical evaluation of lower cut-offs for galactomannan detection in adult neutropenic cancer patients and haematological stem cell transplant recipients. $\mathrm{Br} \mathrm{J}$ Haematol. 2004;126(6):852-860.

7. Wingard JR, White MH, Anaissie E, Raffalli J, Goodman J, Arrieta A; L Amph/ABLC Collaborative Study Group. A randomized, double-blind comparative trial evaluating the safety of liposomal amphotericin B versus amphotericin $\mathrm{B}$ lipid complex in the empirical treatment of febrile neutropenia. L Amph/ABLC Collaborative Study Group. Clin Infect Dis. 2000;31(5):1155-1163.
8. Vehreschild JJ, Sieniawski M, Reuter S, et al. Efficacy of caspofungin and itraconazole as secondary antifungal prophylaxis: analysis of data from a multinational case registry. Int J Antimicrob Agents. 2009;34(5): 446-450.

9. Auberger J, Lass-Flörl C, Aigner M, Clausen J, Gastl G, Nachbaur D. Invasive fungal breakthrough infections, fungal colonization and emergence of resistant strains in high-risk patients receiving antifungal prophylaxis with posaconazole: real-life data from a single-centre institutional retrospective observational study. JAntimicrob Chemother. 2012;67(9):2268-2273.

10. Pagano L, Caira M, Candoni A, et al; SEIFEM Group. Evaluation of the practice of antifungal prophylaxis use in patients with newly diagnosed acute myeloid leukemia: results from the SEIFEM 2010-B registry. Clin Infect Dis. 2012;55(11):1515-1521.

11. Girmenia C, Frustaci AM, Gentile G, et al. Posaconazole prophylaxis during front-line chemotherapy of acute myeloid leukemia: a singlecenter, real-life experience. Haematologica. 2012;97(4):560-567.

12. De Pauw BE, Donnelly JP. Prophylaxis and aspergillosis - has the principle been proven? N Engl J Med. 2007;356(4):409-411.

13. Gomes MZR, Lewis RE, Farias PMCM, Wu C, Kontoyiannis DP. Incidence of breakthrough fungal infection during primary antifungal prophylaxis in acute myeloid leukoemia patients in a cancer centre Abstracts of the 22nd European Congress of Clinical Microbiology and Infectious Diseases, London, UK, March 31-April 3, 2012. Clin Microbiol Infect. 2012;18(Suppl 3);187.

14. Offner F, Cordonnier C, Ljungman P, et al. Impact of previous aspergillosis on the outcome of bone marrow transplantation. Clin Infect Dis. 1998;26(5):1098-1103.

15. Masamoto Y, Nannya Y, Kurokawa M. Voriconazole is effective as secondary antifungal prophylaxis in leukemia patients with prior pulmonary fungal disease: case series and review of literature. J Chemother. 2011;23(1):17-23.

16. Sipsas NV, Kontoyiannis DP. Clinical issues regarding relapsing aspergillosis and the efficacy of secondary antifungal prophylaxis in patients with hematological malignancies. Clin Infect Dis. 2006;42(11): 1584-1591.

17. Nivoix Y, Launoy A, Lutun P, et al. Adherence to recommendations for the use of antifungal agents in a tertiary care hospital. J Antimicrob Chemother. 2012;67(10):2506-2513.

18. Mikulska M, Novelli A, Aversa F, et al. Voriconazole in clinical practice. J Chemother. 2012;24(6):311-327.

19. Park WB, Kim NH, Kim KH, et al. The effect of therapeutic drug monitoring on safety and efficacy of voriconazole in invasive fungal infections: a randomized controlled trial. Clin Infect Dis. 2012;55(8): 1080-1087.

20. Boucher HW, Groll AH, Chiou CC, Walsh TJ. Newer systemic antifungal agents: pharmacokinetics, safety and efficacy. Drugs. 2004;64(18):1997-2020.

21. Hyland R, Jones BC, Smith DA. Identification of the cytochrome P450 enzymes involved in the N-oxidation of voriconazole. Drug Metab Dispos. 2003;31(5):540-547.

22. Pascual A, Calandra T, Bolay S, Buclin T, Bille J, Marchetti O. Voriconazole therapeutic drug monitoring in patients with invasive mycoses improves efficacy and safety outcomes. Clin Infect Dis. 2008;46(2):201-211.

23. Scott LJ, Simpson D. Voriconazole: a review of its use in the management of invasive fungal infections. Drugs. 2007;67(2): 269-298.

24. Metzke B, Neubauer WC, Hieke S, Jung M, Wäsch R, Engelhardt M. Use of systemic antifungals in daily clinical practice in the haematology and oncology setting: results of a prospective observational analysis. Pharmacoepidemiol Drug Saf. 2012;21(9):953-963.

25. Rieger CT, Dittmer M, Ostermann H. [Liposomal amphotericin B in the treatment of severe fungal infections. Results of a clinical cohort trial]. Dtsch Med Wochenschr. 2007;132(40):2062-2066. German.

26. Lebeaux D, Lanternier F, Elie C, et al. Therapeutic drug monitoring of posaconazole: a monocentric study with 54 adults. Antimicrob Agents Chemother. 2009;53(12):5224-5229. 
27. Eksi F, Gayyurhan ED, Balci I. In vitro susceptibility of Candida species to four antifungal agents assessed by the reference broth microdilution method. Scientific World Journal. 2013;2013:236903.

28. Wang $\mathrm{H}$, Xiao M, Chen SC, et al. In vitro susceptibilities of yeast species to fluconazole and voriconazole as determined by the 2010 National China Hospital Invasive Fungal Surveillance Net (CHIF-NET) study. J Clin Microbiol. 2012;50(12):3952-3959.

29. Mokaddas EM, Al-Sweih NA, Khan ZU. Species distribution and antifungal susceptibility of Candida bloodstream isolates in Kuwait: a 10-year study. J Med Microbiol. 2007;56(Pt 2):255-259.

30. Walsh TJ, Gamaletsou MN. Treatment of fungal disease in the setting of neutropenia. Hematology Am Soc Hematol Educ Program. 2013;2013:423-427.
31. van der Linden JW, Snelders E, Kampinga GA, et al. Clinical implications of azole resistance in Aspergillus fumigatus, The Netherlands, 2007-2009. Emerg Infect Dis. 2011;17(10):1846-1854.

32. Iatta R, Caggiano G, Cuna T, Montagna MT. Antifungal susceptibility testing of a 10-year collection of Candida spp. isolated from patients with candidemia. J Chemother. 2011;23(2):92-96.

33. de Fabritiis P, Spagnoli A, Di Bartolomeo P, et al. Efficacy of caspofungin as secondary prophylaxis in patients undergoing allogeneic stem cell transplantation with prior pulmonary and/or systemic fungal infection. Bone Marrow Transplant. 2007;40(3):245-249.

\section{Publish your work in this journal}

Therapeutics and Clinical Risk Management is an international, peerreviewed journal of clinical therapeutics and risk management, focusing on concise rapid reporting of clinical studies in all therapeutic areas, outcomes, safety, and programs for the effective, safe, and sustained use of medicines. This journal is indexed on PubMed Central, CAS,
EMBase, Scopus and the Elsevier Bibliographic databases. The manuscript management system is completely online and includes a very quick and fair peer-review system, which is all easy to use. Visit http://www.dovepress.com/testimonials.php to read real quotes from published authors.

Submit your manuscript here: http://www.dovepress.com/therapeutics-and-clinical-risk-management-journal 